Copyright $\subset 2007$ Associação Brasileira de Pesquisadores em Jornalismo / SBPjor
COMPARATIVE INTERNATIONAL COMMUNICATION PROJECTS: OVERCOMING THE CHALLENGES

\author{
FRANK ESSER \\ University of Mainz, Germany
}

\begin{abstract}
Over the last 10-20 years, comparative research in the field of communication has almost become fashionable. Many factors are responsible for this, for example: an increased awareness of globalisation as a communication-driven process; an awareness of increased transnational conglomerization of media organizations; and the increasing use of the Internet which facilitates easier access to information around the world. But the big question is how to organize collaborative international communication research effectively? Which models of cooperation are available to us, and what are their advantages and disadvantages? In this article, I analyze five ways of doing collaborative researches and their respective challenges.
\end{abstract}

KEY-WORDS

transnational processes; hybridization; comparative research; collaborative research.

\title{
Introduction
}

It is no longer necessary to urge communication scholars to 'go international' in their research. There is now a widespread appreciation of the potential of cross-national collaborations and comparative research. And its potential is vast indeed. The aims include:

- improving understanding of one's own country \& improving understanding of other countries (antidote to ethnocentrism);

- improving international understanding among people and scholars (important means to community building);

- learning from the experiences and initiatives of others (as comparativists, we treat the world as a global laboratory to see what solutions work best under which circumstances) 
- examining transnational processes across different contexts (we study diffusion and globalization processes);

- examining the local reception of imported cultural forms (this refers to concepts like glocalization and hybridization);

- testing a theory across diverse settings (this refers to theory generalization: only comparative research research allows us to assess the generalizability of our theories);

- constructing abstract universally applicable theory (important means to theory building);

- challenging claims to universality (this means that comparative research allows us to see the limits of generalizability and the need to contextualize our theories by taking account of nationand context-specific variables);

- evaluating scope and value of certain phenomena (important strategy for concept clarification and verification);

Over the last 10-20 years, comparative research in the field of communication has almost become fashionable! Many factors are responsible for this, for example: an increased awareness of globalisation as a communication-driven process; an awareness of increased transnational conglomerization of media organizations; and the increasing use of the Internet which facilitates easier access to information around the world.

This last point has changed the work style of us, communication scholars, too. Thanks to internet and email (and thanks to a proliferation of international workshops and conferences), it has become much easier for researchers to exchange ideas and meet more frequently to mull over collaborative projects.

\section{Five models of cooperation}

Our big question is how to organize collaborative international communication research effectively? Which models of cooperation are available to us, and what are their advantages and disadvantages?

The first model for conducting comparative international communication research I would like to examine is what I call the BASIC MODEL. In the basic model, a communication scholar does not want to confine his media research to his home country anymore but wishes to compare it to the media system of another country. 
Here a scholar compares two countries but his research is not truly international because he is hardly able to treat the two countries equally as he will observe the second country (B) through the lens of his own country (A). The home country (A) serves as the backdrop - and often as a yardstick - for examining and evaluating country (B).

This kind of "comparative" research is very frequent because it minimizes many problems unusually associated with cross-national research. You can do it from your home office without leaving your desk; you don't need to bother with a network of collaborators; it seems very efficient and inexpensive. But: it can also be quick and dirty. It can be a dirty approach to comparative research if the researcher does not recognize and acknowledge how deeply embedded our units of analysis usually are in the cultural, historical and social contexts of other countries. Only after many years of travelling and reading pile stocks of books on country $B$, the researcher may achieve a sufficient knowledge to compare units of analysis in both countries adequately and equally. But he will find it difficult to add many more units of analysis to his research, and he will find it even more difficult add a third, fourth, or fifth country to the sample because it would extent his capabilities of accumulating sufficiently large amounts of information on this large number of countries.

One way of overcoming the deficiencies of the Basic Model is a Centralized Model where an international research institution heads comparative projects.

Such "international research centers" which head comparative projects are funded well enough to employ communication scholars from many different countries and to accumulate extensive expertise in foreign media systems among its own rank. Such "centers" are ideal institutions for centrally controlled, competent in-house comparative international research. Unfortunately, there are not too many successful examples of this kind. One example was the "European Media Institute" which does 


\section{(2) Centralized Model of International Research}

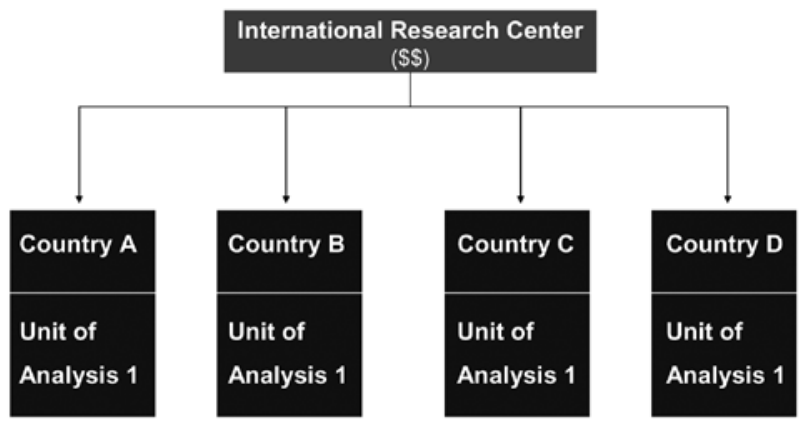

no longer operate as originally devised. It was not able to secure the funds necessary to support its infrastructure and body of researchers for a longer period of time. It would probably be paradise to work in such an international research center, or together with it, but this may also be quite utopian because the centralized model needs substantial third party funding even if there is no research project, and even in times when the research it conducts does not generate any profits.

One possibility of overcoming the disadvantages of the Centralized Model is the Correspondent Models.

\section{(3) Correspondents Model of International Research}

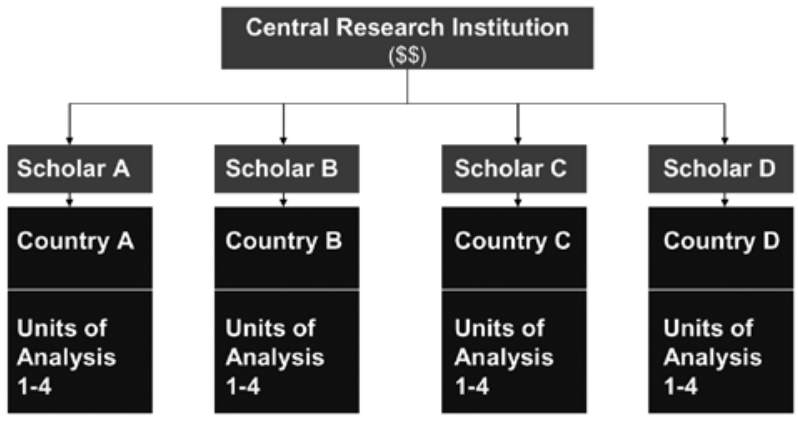


In the correspondent model, we still have a central research institution but it needs much less personnel and infrastructure. The idea here is that a "central institution" (which can be any scholar's office, or any university department, or any non-university institution) obtains funding for a comparative projects (it would usually get the money through a grant) and develops all research tools and data gathering instruments necessary for the project. It also decides autonomously on the theoretical framework for the study, then contacts communication scholars (A-D) in the various countries (A-D) who can be considered experts in the study's main area.

The central institution then treats these experts as national correspondents who are responsible for synchronized data gathering. Synchronized data gathering is achieved by various measures that help ensure that the national correspondents employ all the centralized data gathering instruments (and only those) in their own country and then send back the data to the central headquarters where all data analysis is conducted and where all results are interpreted within the theoretical framework decided on earlier by the people running central headquarters.

This model requires detailed co-ordination and comprehensive control of the work of the national correspondents to achieve equivalence on all levels across all countries. The centralized institution must ensure that the correspondents analyze the units of analysis in identical ways by following centralized guidelines. Because these correspondents work on their home countries they are able to study several units of analysis at the same time, if necessary.

I described the model in rather drastic terms but such a strictly organized shop would actually do the scientific output a lot of good. If successfully executed, this model is capable of generating homogenous, comparable results that are consistently interpreted because all major decisions are made in one central place.

But in the real world, most participating scholars would prefer to work in a more democratic way that also allows for more room for personal research interests. In fact, this model here works best if the correspondents are prepared to take orders from the centralized institute and are prepared participate in a project that leaves little academic freedom. It is built on a clear hierarchy based on centralized funding and centralized planning. 
For all these reasons, in practice this model is realized in much softer, more moderate forms than described here. But even in softer, more moderate forms, this model touches on a few problematic points I would like to share with you. The truth is that it is very likely that the central research institution is located in Northern America or Central Europe where large grants for this kind of research are available.

One of the dirty little secrets of collaborative international research is that the academic world resembles in some ways the center-periphery system Wallerstein described. At the center of the academic world we have a handful of countries in North America and Europe that dominate research. Scholars here are at ease in English and endowed with financial and technical resources. In this small world, they are the ones who could get the research grants, collect and analyze the data, and publish the books and journal articles.

Colleagues at the periphery often have problems finding adequate resources to carry out even modest projects, therefore may lack experience in advanced research techniques, and, often have limited possibilities to produce work that gets accepted for publication in journals controlled by scholars at the center (namely US based academic journals). There are exceptions, as many of us know, but there remains unevenness.

In sum, scholars from the more privileged West are often in the "driver seat" of large comparative international projects, and participating scholars from the periphery are sometimes concerned about what they then perceive as an imperialist attitude of the Western scholars who want to implement their Western structures, theories, methods, and publication strategies in the project (since they brought the money in). It should be pointed out, though, that coming across like imperialists is often the last thing Western scholars want in such a collaborative project but it is important that everybody should be aware of the different perspectives of the project participants.

In particular if participants come from very different parts of the world (West and East, North and South), they may also have different understandings of team work, division of labour, work hierarchies, command structures, information exchange. A more democratic model is the Coordinated Cooperation Model of International Research

It consists of a network of equal scholars (or institutions) from different countries where one scholar serves as "project coordinator". The main difference from the previous model is that all scholars 


\section{(4) Coordinated Cooperation Model of International Research}

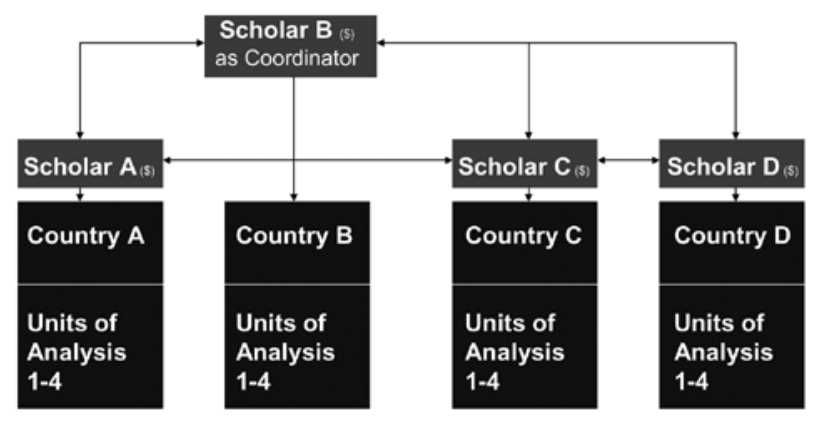

involved participate equally in developing the research design and all research instruments. This type of coordinated cooperation is the kind of research network that is promoted by the European Commission. In order to facilitate research initiatives across member states of the European Union, the European Commission launched a funding framework program 15 years ago that is built on this model.

This model is perhaps the most common in communication research. In its most frequent form, scholars gather at international workshops several times prior to the actual start of the project to agree on all necessary steps. Then, the scholars are responsible for data gathering in their home country, but also for obtaining the necessary funds to carry out the various national projects. Because scholars know their home country very well more complex research designs with several units of analysis can be realized.

The biggest problem here is that participating researchers have to find agreement on everything voluntarily which can be difficult for many reasons. In this model, often enough, researchers find themselves comparing not only their findings but also their theories and concepts, methodological preferences, research ethics, writing styles and publication strategies. And as mentioned before, crossnational variations in professional academic cultures can create massive difficulties.

In terms of soft skills, it's important that participating researchers are able to sustain good working relationships at a distance and over a considerable time, relying heavily on communication etiquette and 
conventions of trust and courtesy. Some participating researchers may suffer difficulties with writing and working in a foreign language; some may suffer inequalities in funding \& institutional support; other may even experience anxieties over the issues of data ownership and intellectual property that arise in collaboration. ' All these points need to be addressed ahead of time to avoid irritation.

In terms of more substantial scientific problems, participating researchers have to find a solution for how to deal with different academic cultures and scientific socializations. Too much theoretical diversity can seriously threaten a collaborative project. The first strategy to deal with theoretical diversity is a centralized strategy where one theory is decided on and all potential alternatives are being disregarded (Swanson called this the avoidance strategy). The second path is a theory-neutral strategy where data gathering is planned without any guiding theory and the theory-work only enters the picture much later when the results are being interpreted (Swanson called this the pre-theoretical strategy). The third strategy is the most common denominator approach where the project is guided by a very general, very broad theoretical orientation that serves as an umbrella for very divergent scientific approaches (Swanson: metatheoretical strategy). In my own personal experience, agreement on what theories to use has always been a difficult decision and involved always time-consuming debates.

The "Coordinated Cooperation Model" is the prerequisite for the last and most advanced cooperation model - the Coordinated, Fully Comparative Cooperation Model. This model refers to the data analysis stage after all data has been gathered in the different countries. In the data gathering stage, each scholar examines his own home country but in the data analysis stage, each scholar works with the entire data set and analyses research questions that involve all the countries in the project. This way, it becomes a fully comparative project. Every participating scholar examines a different unit of analysis (or research question) and compares all countries on this aspect. It is the most advanced model because it requires the various scholars to have sufficient background knowledge on all participating countries to reach meaningful interpretations of the results.

This model of cooperation is actually more common in the natural sciences than in the field of communication because media phenomena tend to be strongly determined by culture and language so that only rarely scholars will be confident enough to draw competent 


\section{(5) Coordinated, Fully Comparative Cooperation Model}

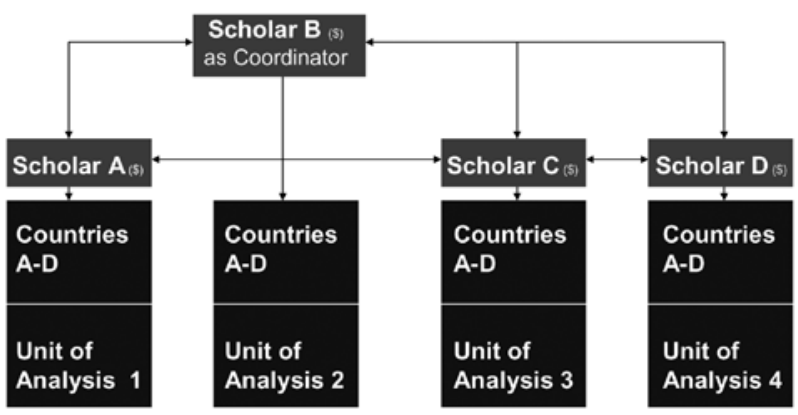

comparisons across so many nations and cultures.

Another problem besides lack of knowledge is lack of interest. The idea that scholars from around the world would collectively interrogate the data often fails because scholarship, like news, is local. Mostly, scholars are mainly interested in interpreting their own national data, and publishing it locally.

A third problem is that co-operative projects require frequent and extensive meetings for scholarly exchange and mutual planning. But the reality is that when we meet at international conferences, time is short and the agenda crowded, and we are usually not there long enough to clear the fog of jet lag. Intense, extended intellectual discussion at conferences is rare - and time and money for additional workshops is usually not sufficiently available.

\section{Conclusion}

How can we summarize the lessons for novices interested in collaborative work? Read international journals and attend international conferences in an effort to find scholars who share your research interests. If you decide on an international collaboration (for the reasons spelt out on slide 1), agree on a model of cooperation (which is often determined by the funding situation):

- Agree on theory! Agree on methods!

- Helpful project principle 1: "Give a little, get a lot."

- Helpful project principle 2: "Seek collective publication strategy" 
- Beware of the worst case scenario

- Strive for the best case scenario.

The project principles, "Give a little, get a lot", means that in return for contributing a small amount of data on one's own country, the individual scholar is promised the complete data set. The hope is that individual participants would find creative ways to explore the data and to test hypotheses beyond anything possible in data from a single country.

The second project principle is a promise to "seek collective publication" of results while encouraging participants to publish independently on their own. For some, an individually authored chapter in a book published in Europe or the United States was an important professional achievement. For others, publications locally and in the local language were more important.

The "worst case scenario" is that the effort to coordinate the project is to time- and energy consuming that the projects never gets fully completed and fully published (a fate suffered by several collaborative international projects in our field). What adds to this scenario is that so many compromises between the participants (theory and methodwise) can be necessary that the results are so unoriginal and noninnovative that they cannot compete with the state of the art in the field - and therefore a large international project may well go down in history without leaving any impact on the discipline.

The "ideal case scenario" is that - based on mutual exchanges between international academic communities and supported by national and international associations - strong ties of collaborative cooperation in teaching, research and exchange programs emerge which set the frame for cross-cultural projects that are sufficiently funded to cover all costs for research, administration and travel, and which address socially relevant questions and generate intriguing results that leave a deep impact on the field.

\section{NOTE}

1 In short, comparative work relies not only on time, funding and mutual interest, but also on good will, on trust and 'emotional labour'. In fact, this model of cooperative collaboration confuses the boundary between the professional and the personal - to borrow an argument from Sonia Livingstone. This model can only succeed if the researchers involved become, to some degree, friends. 
Frank Esser is assistant professor in the Institut fuer Publizistik, in University of Mainz, Germany. He holds a PhD degree in Economics Sciences from University of Mainz and has been a visiting lecturer at other universities, in Europe and North America. Currently, he is serving as the International Affairs Coordinator of the German Communication Association. In his work Esser focuses on mass communication from a cross-national perspective.

Email: esserf@missouri.edu 